\title{
Paternal Subclinical Hypothyroidism Affects the Clinical Outcomes of In Vitro Fertilization/Intracytoplasmic Sperm Injection
}

\author{
Meng Rao, Zexing Yang, ${ }^{1}$ Cunmei Su, Zihan Zhao, Ruixue Wan, Jiang Liu, \\ Youlin Yao, ${ }^{1}$ Zhenfang Su, ${ }^{1}$ Kunhua Wang, ${ }^{2}$ Li Tang, ${ }^{1}$ and Shuhua Zhao ${ }^{1}$
}

Background: Maternal subclinical hypothyroidism $(\mathrm{SCH})$ is a risk factor for adverse pregnancy outcomes. However, it is still unclear whether SCH affects male fertility. The aim of this study was to determine the association between paternal SCH and clinical outcomes after in vitro fertilization/intracytoplasmic sperm injection (IVF/ICSI).

Methods: This retrospective study included 2511 couples with paternal euthyroidism $(n=2282)$ or SCH $(n=229)$ who visited our clinic for infertility treatment between April 1, 2017, and September 30, 2019. The primary outcomes were the fertilization rate and clinical pregnancy rate; the secondary outcomes were the good-quality embryo rate, blastocyst formation rate, implantation rate, and early miscarriage rate. These outcomes were compared between the euthyroid and the $\mathrm{SCH}$ groups after adjusting for various potential confounders.

Results: The mean paternal ages in the euthyroid and SCH groups were 34.5 and 36.0 years, respectively $(p=0.002)$. Semen parameters and sperm DNA fragmentation index were similar between the two groups (all $p>0.05)$. The adjusted fertilization ( 0.69 vs. $0.71, p=0.30)$, good-quality embryo $(0.49$ vs. $0.52, p=0.31)$, blastocyst formation ( 0.51 vs. $0.53, p=0.57$ ), and early miscarriage ( 0.11 vs. $0.10, p=0.81)$ rates were also similar between the two groups. There was a significantly decreased adjusted clinical pregnancy rate [confidence interval, $\mathrm{CI}]$ and implantation rate $[\mathrm{CI}]$ in the paternal SCH group compared with the euthyroid group $(0.32[0.26-0.40]$ vs. $0.42[0.40-0.45], p=0.009$ for the clinical pregnancy rate; 0.24 [0.19-0.29] vs. 0.29 [0.27-0.31], $p=0.037$ for the implantation rate). Stratified analysis indicated that these differences were only significant in men aged $\geq 35$ years ( $p=0.009$ and 0.022 , respectively) and not in men $<35$ years ( $p=0.39$ and 0.45 , respectively).

Conclusions: Paternal SCH was associated with worse clinical outcomes after IVF/ICSI, whereas this detrimental impact was only present in males $\geq 35$ years old. Prospective studies and basic research are warranted to confirm these results and to clarify the mechanisms underlying these associations, respectively.

Keywords: subclinical hypothyroidism, thyroid function, IVF/ICSI, clinical outcomes

\section{Introduction}

$\mathbf{S}$ UBCLINICAL HYPOTHYROIDISM (SCH) is defined as an elevated serum thyrotropin (TSH) concentration coexisting with a normal serum thyroxine (T4) concentration. In our previous study, we identified an association between maternal SCH and decreased ovarian reserve (1). Others have also described associations between maternal SCH and adverse pregnancy outcomes, such as a decreased good-quality embryo rate or clinical pregnancy rate, or an increased risk of miscarriage (2-5). Women with SCH might therefore benefit from levothyroxine (LT4) supplementation before or during their attempt to conceive (6). However, the effect of LT4 supplementation on naturally conceived pregnancies and pregnancies achieved by assisted reproductive technology (ART) is different: LT4 supplementation significantly decreases the odds of pregnancy loss in pregnancies achieved by ART, but not in naturally conceived pregnancies, among women with SCH. By contrast, LT4 seems to reduce the risks of pregnancy loss and preterm birth in naturally conceived pregnancies, but not in pregnancies achieved by ART, among patients with thyroid autoimmunity (TAI), as indicated by our previously published study (6). These different effects suggest that SCH may influence the fertility of infertile

${ }^{1}$ Department of Reproduction and Genetics, The First Affiliated Hospital of Kunming Medical University, Kunming, China.

${ }^{2}$ The NHC Key Laboratory of Drug Addiction Medicine, The First Affiliated Hospital of Kunming Medical University, Kunming, China. 
women in a different way from fertile women. Although thyroid hormones (THs) are important in regulating fertility in women, their effects on fertility in men are largely unknown (7).

In the male reproductive system, germ cells, Sertoli cells, and Leydig cells express TH receptors (THRs) (8-11). THs regulate testis function and spermatogenesis by binding to these THRs to form hormone-receptor complexes, which subsequently activate gene transcription and protein synthesis $(8,10)$. THs also enhance cyclic adenosine monophosphate synthesis and $\mathrm{Ca}^{2+}$ release in spermatozoa. This process enhances flagellar movement and ultimately improves sperm motility $(11,12)$.

$\mathrm{SCH}$ represents a subtle deficiency in thyroid function despite the fact that $\mathrm{TH}$ concentrations remain within the normal range. Whether such mild thyroid dysfunction in men can affect the clinical outcomes of assisted reproduction is unclear. Here we aimed to investigate the potential effects of paternal SCH on the clinical outcomes of in vitro fertilization/intracytoplasmic sperm injection (IVF/ICSI).

\section{Methods}

\section{Study approval and informed patient consent}

This study was approved by the First Affiliated Hospital of Kunming Medical University, China. All patients provided signed informed consent for the use of personal clinical data in this study before enrolling in an ART treatment cycle.

\section{Study design}

This retrospective study was conducted at the Reproductive Medical Center of the First Affiliated Hospital of
Kunming Medical University between April 1, 2017, and September 30, 2019. All patients who were evaluated at this fertility clinic were offered screening examinations on the first visit to determine whether further ART treatments were suitable. For men, these examinations were of semen, sperm morphology, sperm DNA fragmentation, and thyroid function. For women, these examinations included transvaginal ultrasonography to determine the antral follicle count (AFC) and biochemical analyses to determine the anti-Müllerian hormone (AMH) concentration, reproductive hormone concentrations on days 2-4 of the menstrual cycle, and thyroid function. All participants were screened for hepatitis, human immunodeficiency virus, and syphilis infection. The demographic and clinical data pertaining to each patient who underwent ART were recorded in a database.

\section{Inclusion and exclusion criteria}

Potentially eligible couples (cycles) were included if (i) they were undergoing the first IVF or ICSI cycle with autologous sperm and oocytes; (ii) the male partner had either normal thyroid function or $\mathrm{SCH}$; and (iii) the female partner had normal thyroid function. Couples were excluded if (i) either partner had a history of thyroid disease (treated or not); (ii) either partner had a tumor or a systematic disease, such as hypertension, diabetes, or another endocrine disease; (iii) either partner had chromosomal abnormalities; (iv) the female partner had a uterine abnormality; or (v) no oocytes were aspirated during oocyte retrieval. Finally, 2511 couples were enrolled in the study and their demographic and clinical data were collected for analysis. These couples comprised 2282 clinically euthyroid men and 229 men diagnosed with $\mathrm{SCH}$ (Fig. 1).

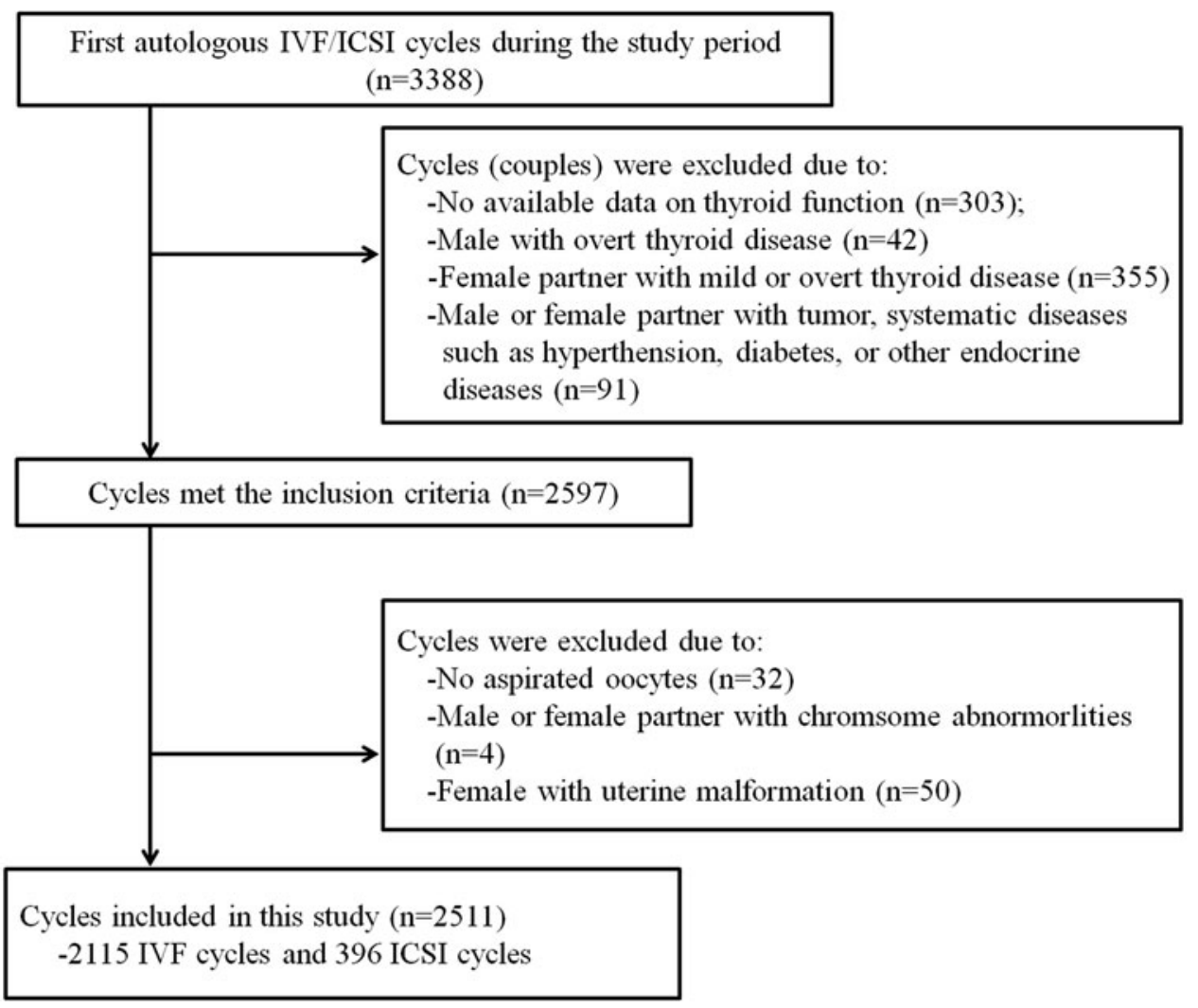

FIG. 1. Flowchart for final study population selection. 


\section{AFC measurements}

The ovarian AFCs were determined on days 2-4 of an unstimulated menstrual cycle. The AFC was defined as the sum of the antral follicles $(2-9 \mathrm{~mm}$ in diameter) in both ovaries as measured by transvaginal ultrasound during the early follicular phase. All AFC evaluations were performed by the same two highly trained reproductive endocrinologists, as previously described (13).

\section{Thyroid function and $\mathrm{AMH}$ concentration measurements}

The serum concentrations of TSH, free triiodothyronine (fT3), free thyroxine (fT4), thyroperoxidase antibody (TPO$\mathrm{Ab})$, and thyroglobulin antibody $(\mathrm{Tg}-\mathrm{Ab})$ were measured by automated chemiluminescent immunoassay using a Cobas E601 analyzer (Roche Diagnostics GmbH, Mannheim, Germany), as previously described $(14,15)$. Commercial analytic kits were also purchased from Roche Diagnostics $\mathrm{GmbH}$. The serum AMH concentration was assayed using a commercially available ELISA kit (Kangrun Biotech, Guangzhou, China) with a sensitivity of $0.06 \mathrm{ng} / \mathrm{mL}$, and the data were read on a plate reader (DS2; Dynex Technologies, Chantilly, VA), as previously described (16). The following reference ranges were recommended by the manufacturer: $0.27-4.20$ $\mathrm{IU} / \mathrm{mL}$ for TSH, 3.1-6.8 pmol/L for fT3, 12-22 pmol/L for fT4, <115.0 IU/mL for Tg-Ab, and $<34 \mathrm{IU} / \mathrm{mL}$ for TPO-Ab. A diagnosis of SCH was made if the TSH concentration was $>4.2 \mathrm{mIU} / \mathrm{mL}$, but the fT 4 concentration remained within the reference range. A diagnosis of TAI was made if either the $\mathrm{TPO}-\mathrm{Ab}$ concentration was $>34 \mathrm{IU} / \mathrm{mL}$ or the $\mathrm{Tg}-\mathrm{Ab}$ concentration was $>115 \mathrm{IU} / \mathrm{mL}$.

\section{Semen analysis}

Semen samples were collected by masturbation after two to seven days of sexual abstinence. After liquefaction at $37^{\circ} \mathrm{C}$, the samples were examined for the semen volume and the sperm concentration, motility, and morphology. The semen volume was determined from the semen weight, assuming a density of $1.0 \mathrm{~g} / \mathrm{mL}$.

To assess the sperm concentration and motility, a $10-\mu \mathrm{L}$ sample of well-mixed semen was placed in a clean Makler chamber (prewarmed to $37^{\circ} \mathrm{C}$ ), and the sample was covered with a coverslip and immediately examined using a Sperm Class Analyzer (SCA-P-H-02; Microptic S.L., Spain), as previously described (17).

Sperm morphology was evaluated according to the World Health Organization (WHO) criteria, Fifth Edition (18). Briefly, a $10-\mu \mathrm{L}$ sample of well-mixed semen was placed on a slide to prepare a smear. The sperm were fixed and stained by the Diff-Quick method. All steps were performed using a commercial kit, according to the manufacturer's instructions (Huakang Biomed Ltd., Shenzhen, China). The morphologies of 200 spermatozoa were evaluated under a microscope (CX23; Olympus, Tokyo, Japan) and the proportion of morphologically normal sperm was calculated, as previously described (17). All semen sample analyses were performed by the same highly trained laboratory technician using the same apparatus. According to the WHO criteria (18), semen samples with the following parameters were classified as normal: semen volume $\geq 1.5 \mathrm{~mL}, \mathrm{pH} \geq 7.2$, sperm concentra- tion $\geq 15 \times 10^{6} / \mathrm{mL}$, progressive motility $\geq 32 \%$, and normal morphology rate $\geq 4 \%$.

\section{Sperm DNA fragmentation determination}

Sperm DNA fragmentation was evaluated using a sperm chromatin dispersion test, according to the manufacturer's instructions (Huakang Biomed Ltd.) and as previously described (19). Briefly, a $60-\mu \mathrm{L}$ diluted semen sample $\left(5-10 \times 10^{6} / \mathrm{mL}\right)$ was added to $1 \%$ low-melting-point aqueous agarose that had been prewarmed for 5 minutes in a $90-100^{\circ} \mathrm{C}$ water bath. After careful mixing, $30 \mu \mathrm{L}$ of this mixture was placed onto a super-coated slide (provided with the kit) and incubated at $4^{\circ} \mathrm{C}$ for 4 minutes. The slide was then successively immersed in solution $\mathrm{A}$ and solution $\mathrm{B}$, and then dehydrated using an ethanol gradient. Finally, the slide was treated with Wright staining solution and air dried. A minimum of 400 spermatozoa were observed under a microscope and scored according to the patterns established by Fernandez et al. (20). The proportion of spermatozoa with DNA fragmentation was defined as the DNA fragmentation index (DFI).

\section{Ovarian stimulation, oocyte aspiration, and embryo transfer}

Women were subjected to different ovarian stimulation protocols, including the long GnRH agonist (long protocol, $n=1280$ ), GnRH antagonist (antagonist protocol, $n=762$ ), and mild stimulation protocols $(n=441)$, as well as other protocols, including 25 natural cycles and 28 progestinprimed ovarian stimulation cycles. The stimulation protocol was selected based on the health condition of the patient, as previously described (21-23). The long agonist protocol: An adjusted dose (1.00-3.75 mg) of triptorelin (Decapeptyl ${ }^{\circledR}$; Ipsen Pharma Biotech, Paris, France) was administered according to the patient's weight and body mass index (BMI). The antagonist protocol: A GnRH antagonist (Cetrotide ${ }^{\circledR}$; Serono, Geneva, Switzerland) was used at a dosage of $0.25 \mathrm{mg} /$ day. The progestin-primed ovarian stimulation protocol: Medroxyprogesterone acetate or dihydrogesterone was used to suppress a premature luteinizing hormone surge during the follicular phase, as previously described (24). The clinician determined the optimal timing and dosage of gonadotropin (Gonal-F ${ }^{\circledR}$; Merck Serono, Geneva, Switzerland) based on B-ultrasound images and serum hormone concentrations. Recombinant human chorionic gonadotropin (HCG, $250 \mathrm{mg}$, Ovidrel; Serono) was administered when the two leading follicles reached a mean diameter of $18 \mathrm{~mm}$.

Oocytes were retrieved transvaginally, 34-36 hours after HCG administration, and the follicles were aspirated under transvaginal ultrasound guidance using a single lumen needle attached to a syringe. Semen was prepared by density gradient centrifugation technique, and insemination was carried out by conventional IVF or ICSI. Oocyte fertilization was assessed 18-20 hours after insemination by confirming the presence and location of two pronuclei. The embryos were graded on a scale from 1 to 4 according to the number, size, and shape of the blastomeres and the degree of fragmentation (25). Grade I and II embryos were defined as being of good quality; these embryos were prioritized for transfer. Grade III embryos were only transferred if no grade I or II embryos were available. Blastocyst development was assessed on day 5-6 after considering the degree of expansion and the 
appearance of an inner cell mass and trophectoderm. Up to three fresh or frozen embryos were transferred, depending on the patient's condition.

\section{Outcome measures}

The primary outcome measures in this study were the fertilization rate and the clinical pregnancy rate. The secondary outcome measures were the good-quality embryo rate, blastocyst formation rate, implantation rate, and early miscarriage rate. The fertilization rate was calculated as the number of normally fertilized oocytes (two pronuclei) divided by the total number of oocytes inseminated (IVF cycle) or as per the total number of oocytes injected (ICSI cycle). The good-quality embryo rate was calculated as the proportion of grade I and II embryos among all embryos evaluated on day 3 . The blastocyst formation rate was calculated as the number of blastocysts divided by the number of embryos that underwent blastocyst culture. Clinical pregnancy was defined as the identification of a gestational sac with fetal heart activity on ultrasound examination four to five weeks after embryo transfer. The implantation rate was calculated as the number of gestational sacs (observed four weeks after transfer) divided by the number of embryos transferred. The early miscarriage rate was calculated as the number of cycles with pregnancy loss before 12 weeks of gestation divided by the number of cycles resulting in clinical pregnancy.

\section{Sample size calculation}

According to previous data analysis, the prevalence of $\mathrm{SCH}$ in men attending the Reproductive Medical Center was $\sim 9 \%$ and the proportion of paternal SCH to paternal euthyroidism was $\sim 1: 10$. Based on these data, a sample size of 2511 couples (2282 with euthyroidism and $229 \mathrm{SCH}$ ) was determined to be sufficient to demonstrate a $10 \%$ difference in the clinical pregnancy rate between the two groups, with $85 \%$ power and a $5 \%$ significance concentration $(\alpha)$. The sample size calculation was performed using PASS 11 software (NCSS, UT).

\section{Statistical analyses}

Continuous variables are presented as the means \pm standard deviations when normally distributed or as medians (interquartile ranges). A Mann-Whitney $U$ test (continuous variables) or chi-square test (categorical variables) was used to identify differences in the demographic and clinical data between euthyroid men and men with $\mathrm{SCH}$.

The influence of SCH on semen parameters and IVF/ICSI outcomes was analyzed using generalized linear models. To analyze the semen parameters, the model was adjusted for the following confounders in men: age (continuous), BMI (continuous), TAI (categorical), smoking status (categorical), alcohol consumption (categorical), and the duration of abstinence (continuous). To analyze the laboratory outcomes (i.e., the fertilization rate, good-quality embryo rate, and blastocyst formation rate), the models were adjusted for male age, BMI, TAI, smoking status, and alcohol consumption; female age, BMI, and TAI; and primary/secondary infertility (categorical), an infertility diagnosis (categorical), the number of aspirated oocytes (continuous), the ovarian stimulation protocol (categorical), and IVF or ICSI cycles (categorical).
To analyze clinical outcomes (i.e., the clinical pregnancy rate, implantation rate, and early miscarriage rate), the models were additionally adjusted for fresh/frozen cycles (categorical), the number of transferred embryos (categorical), and the day of embryo transfer (day 3 or 5-6) (categorical).

A regression analysis stratified by age ( $<35$ and $\geq 35$ years) was performed to investigate whether the association between SCH and IVF/ICSI outcomes differed between younger and older men. To evaluate whether paternal TAI affected the IVF outcome, the patients were further subdivided into four groups: euthyroidism, isolated $\mathrm{SCH}$, isolated TAI, and $\mathrm{SCH}$ complicated with TAI (SCH+TAI). The clinical outcomes between these groups were also compared and the potential confounders were adjusted to the above models.

All statistical tests were two sided and a $p$-value $<0.05$ was considered statistically significant. SPSS 25.0 (SPSS, Inc., Chicago, IL) was used for the data analysis.

\section{Results}

\section{The characteristics of the study cohort}

This study included 2511 couples who underwent their first IVF/ICSI cycle and met the defined study inclusion and exclusion criteria. Of these couples, 2282 and 229 were classified into the paternal euthyroid and $\mathrm{SCH}$ groups, respectively. The mean age of the men (and their respective partners) in the SCH group was greater than the mean age of the men in the euthyroidism group (male age: 36.0 years vs. 34.5 years, $p=0.002$; female age: 33.6 years vs. 32.5 years, $p=0.01$, respectively). The mean BMI for the men (and their respective partners) was similar between both groups ( $p=0.17$ and 0.89 , respectively). Men with $\mathrm{SCH}$ had a significantly lower fT4 concentration than euthyroid men (median value: $17.35 \mathrm{pmol} / \mathrm{L}$ vs. $18.00 \mathrm{pmol} / \mathrm{L}, p<0.001)$. One hundred thirty-two of the $2282(5.78 \%)$ men in the euthyroidism group had TAI, whereas 32 of the 229 men $(13.97 \%)$ with SCH had TAI $(p<0.001)$. The frequency of alcohol consumption and smoking in men was similar between the two groups. We observed no significant intergroup differences in the duration of abstinence, semen volume, sperm concentration, sperm morphology, or DFI apart from a slight but significantly lower progressive sperm motility in the paternal SCH group compared with the paternal euthyroid group (35.0\% vs. $37.6 \%, p=0.04$; Table 1$)$.

\section{No association between $\mathrm{SCH}$ and semen parameters}

After adjusting for potential confounders, we found no significant differences in the semen volume, sperm concentration, progressive sperm motility, sperm morphology, or DFI between the paternal euthyroid and $\mathrm{SCH}$ groups (all $p>0.05$ ) (Table 2).

\section{The associations between paternal SCH and ART outcomes in couples}

The adjusted fertilization rate [confidence interval, $\mathrm{CI}$ ] in the paternal $\mathrm{SCH}$ group was similar to that in the paternal euthyroidism group (0.69 [0.66-0.73] vs. 0.71 [0.70-0.72], $p=0.30)$. The adjusted good-quality embryo (0.49 [0.45$0.54]$ vs. 0.52 [0.50-0.53], $p=0.31)$ and blastocyst formation $(0.51[0.46-0.56]$ vs. $0.53[0.51-0.54], p=0.57)$ rates were also similar between these two groups. Nevertheless, the 
Table 1. Characteristics of Study Cohort

\begin{tabular}{|c|c|c|c|}
\hline Characteristics & Euthyroidism $(\mathrm{n}=2282)$ & $S C H(\mathrm{n}=229)$ & $\mathrm{p}$ \\
\hline \multicolumn{4}{|l|}{ Male } \\
\hline Age, years, mean (SD) & $34.5(6.2)$ & $36.0(6.8)$ & 0.002 \\
\hline $\mathrm{BMI}, \mathrm{kg} / \mathrm{m}^{2}$, mean $(\mathrm{SD})$ & $24.2(3.3)$ & $24.5(3.2)$ & 0.17 \\
\hline \multicolumn{4}{|l|}{ Smoking status, $n(\%)$} \\
\hline Never & $1078(47.2)$ & $116(50.7)$ & \multirow[t]{3}{*}{0.52} \\
\hline Current & $541(23.7)$ & $54(23.6)$ & \\
\hline Former & $663(29.1)$ & $59(25.8)$ & \\
\hline \multicolumn{4}{|l|}{ Alcohol consumption, $n(\%)$} \\
\hline Never & $1002(43.9)$ & $108(47.2)$ & \multirow[t]{3}{*}{0.57} \\
\hline Current & $601(26.3)$ & $54(23.6)$ & \\
\hline Former & $679(29.8)$ & $67(29.3)$ & \\
\hline TSH, mIU/mL, median (IQR) & $2.07(1.27)$ & $5.27(1.45)$ & $<\mathbf{0 . 0 0 1}$ \\
\hline fT3, pmol/L, median (IQR) & $5.22(0.80)$ & $5.22(0.86)$ & 0.45 \\
\hline $\mathrm{fT} 4, \mathrm{pmol} / \mathrm{L}$, median (IQR) & $18.00(3.16)$ & $17.35(3.69)$ & $<\mathbf{0 . 0 0 1}$ \\
\hline \multicolumn{4}{|l|}{ TAI, $n(\%)$} \\
\hline Positive & $132(5.8)$ & $32(14.0)$ & \multirow[t]{2}{*}{$<\mathbf{0 . 0 0 1}$} \\
\hline Negative & $2150(94.2)$ & $197(86.0)$ & \\
\hline \multicolumn{4}{|l|}{ Semen parameters } \\
\hline Duration of abstinence, day, median (IQR) & $4.0(4.0)$ & $5.0(4.0)$ & 0.17 \\
\hline Semen volume, $\mathrm{mL}$, median (IQR) & $3.2(2.1)$ & $3.2(2.2)$ & 0.44 \\
\hline Sperm concentration, $10^{6} / \mathrm{mL}$, median (IQR) & $72.4(67.8)$ & $78.9(65.7)$ & 0.26 \\
\hline Progressive sperm motility, \%, median (IQR) & $37.6(18.5)$ & $35.0(21.2)$ & 0.04 \\
\hline Sperm morphology, \%, median (IQR) & $7.0(3.0)$ & $6.0(3.0)$ & 0.44 \\
\hline DFI, median (IQR) & $18.0(8.0)$ & $18.0(11.0)$ & 0.12 \\
\hline \multicolumn{4}{|l|}{ Female } \\
\hline Age, years, mean (SD) & $32.5(5.7)$ & $33.6(6.2)$ & 0.01 \\
\hline BMI, $\mathrm{kg} / \mathrm{m}^{2}$, mean $(\mathrm{SD})$ & $22.6(3.3)$ & $22.6(3.4)$ & 0.89 \\
\hline \multicolumn{4}{|l|}{$\mathrm{AMH}, \mathrm{ng} / \mathrm{mL}, n(\%)$} \\
\hline$<1.5$ & $542(23.8)$ & $66(28.8)$ & \multirow[t]{2}{*}{0.09} \\
\hline$\geq 1.5$ & $1740(76.2)$ & $163(71.2)$ & \\
\hline \multicolumn{4}{|l|}{$\mathrm{AFC}, n(\%)$} \\
\hline$<7<$ & $389(17.0)$ & 49 (21.4) & \multirow[t]{2}{*}{0.10} \\
\hline$\geq 7$ & $1893(83.0)$ & $180(78.6)$ & \\
\hline \multicolumn{4}{|l|}{ TAI, $n(\%)$} \\
\hline Positive & $231(10.1)$ & $28(12.2)$ & \multirow[t]{2}{*}{0.32} \\
\hline Negative & $2051(89.9)$ & $201(87.8)$ & \\
\hline \multicolumn{4}{|l|}{ Type of infertility, $n(\%)$} \\
\hline Primary & $892(39.1)$ & $83(36.2)$ & 0.40 \\
\hline Secondary & $1390(60.9)$ & $146(63.8)$ & \\
\hline Infertility diagnosis, $n(\%)$ & & & \\
\hline Tubal factor & 1359 (59.6) & $134(58.5)$ & 0.51 \\
\hline Diminished ovarian reserve & $320(14.0)$ & $41(17.9)$ & \\
\hline Male factor & $225(9.9)$ & $21(9.2)$ & \\
\hline Ovulation dysfunction & $121(5.3)$ & $14(6.1)$ & \\
\hline Unexplained & $85(3.7)$ & $6(2.6)$ & \\
\hline Both factors & $172(7.5)$ & $13(5.7)$ & \\
\hline Ovarian stimulation protocol, $n(\%)$ & & & \\
\hline Long protocol & $1175(51.5)$ & $105(45.9)$ & 0.16 \\
\hline Antagonist protocol & $689(30.2)$ & 73 (31.9) & \\
\hline Mild stimulation protocol & $368(16.1)$ & $48(21.0)$ & \\
\hline Other protocols & $50(2.2)$ & $3(1.3)$ & \\
\hline No. of aspirated oocytes, median (IQR) & $10(11)$ & $9(12)$ & 0.11 \\
\hline Day of embryo transfer, $n(\%)$ & & & \\
\hline Day 3 & $1450(71.3)$ & $143(72.2)$ & 0.77 \\
\hline Day $5 / 6$ & $585(28.7)$ & $55(27.8)$ & \\
\hline Embryo transfer, $n(\%)$ & & & \\
\hline Fresh & $886(43.5)$ & 79 (39.9) & 0.32 \\
\hline Frozen & $1149(56.5)$ & $119(60.1)$ & \\
\hline No. of transferred embryos, $n(\%)$ & & & \\
\hline 1 & $395(19.4)$ & $46(23.2)$ & 0.35 \\
\hline 2 & $1635(80.3)$ & $152(76.8)$ & \\
\hline 3 & $5(0.3)$ & $0(0)$ & \\
\hline
\end{tabular}

Bold $p$-values indicate a significant difference.

AFC, antral follicle count; AMH, anti-Müllerian hormone; BMI, body mass index; DFI, DNA fragmentation index; fT3, free triiodothyronine; fT4, free thyroxine; IQR, interquartile range; $\mathrm{SCH}$, subclinical hypothyroidism; SD, standard deviation; TAI, thyroid autoimmunity; TSH, thyrotropin. 
Table 2. Associations Between Subclinical Hypothyroidism and Semen Parameters (Presented as Adjusted Mean [Confidence Interval])

\begin{tabular}{lccc}
\hline Semen parameters & Euthyroidism $(\mathrm{n}=2282)$ & $S C H(\mathrm{n}=229)$ & $\mathrm{p}$ \\
\hline Semen volume, $\mathrm{mL}$ & $3.5[3.4-3.6]$ & $3.4[3.2-3.6]$ & 0.52 \\
Sperm concentration, $10^{6} / \mathrm{mL}$ & $84.0[81.7-86.3]$ & $84.6[77.3-91.8]$ & 0.88 \\
Progressive sperm motility, \% & $37.5[36.9-38.2]$ & $36.2[34.3-38.1]$ & 0.20 \\
Sperm morphology, \% & $6.7[6.6-6.8]$ & $6.7[6.4-7.0]$ & 0.69 \\
DFI, \% & $18.7[18.4-19.0]$ & $19.7[18.8-20.6]$ & 0.06 \\
\hline
\end{tabular}

Adjusted for male age, BMI, TAI, smoking status, alcohol consumption, and duration of abstinence.

adjusted clinical pregnancy rate in the paternal $\mathrm{SCH}$ group was significantly lower than that in the paternal euthyroidism group (0.32 [0.26-0.40] vs. 0.42 [0.40-0.45], $p=0.009)$. The implantation rate in the paternal $\mathrm{SCH}$ group was also significantly lower than that in the paternal euthyroidism group (0.24 [0.19-0.29] vs. 0.29 [0.27-0.31], $p=0.037)$. We observed no significant difference in the early miscarriage rate between these two groups $(p=0.81)$ (Table 3$)$.

The associations between paternal SCH and ART outcomes stratified by paternal age

To investigate whether paternal age affects the association between paternal SCH and ART outcomes, we performed a subgroup analysis after stratifying the patients by male age ( $<35$ and $\geq 35$ years). Among men aged $<35$ years, we found a slight but nonsignificant decrease in the fertilization rate, good-quality embryo rate, blastocyst formation rate, clinical pregnancy rate, and implantation rate in the paternal $\mathrm{SCH}$ group compared with the paternal euthyroidism group (all $p>0.05$ ). Among men aged $\geq 35$ years, we also found a slight but nonsignificant decrease in the fertilization rate, goodquality embryo rate, and blastocyst formation rate in the paternal SCH group compared with the paternal euthyroidism group (all $p>0.05$ ). However, the clinical pregnancy and implantation rates in the paternal $\mathrm{SCH}$ group were significantly lower than the equivalent rates in the paternal euthyroidism group (adjusted value [CI] 0.18 [0.11-0.29] vs. $0.31[0.27-0.35], p=0.009$ for the clinical pregnancy rate; $0.13[0.08-0.21]$ vs. $0.21[0.18-0.23], p=0.022$ for the im- plantation rate). The early miscarriage rate showed a slight but nonsignificant increase in the paternal $\mathrm{SCH}$ group compared with the paternal euthyroidism group (0.18 [0.05-0.46] vs. $0.15[0.11-0.21], p=0.81)$ (Table 4$)$.

\section{The associations between paternal $\mathrm{SCH}$ and $A R T$ outcomes stratified by IVF/ICSI cycles}

We also evaluated the effect of paternal SCH on ART outcomes according to IVF and ICSI cycles. Of the 2115 IVF cycles, 1916 and 199 men were characterized by euthyroidism and $\mathrm{SCH}$, respectively. The adjusted clinical pregnancy rate $[\mathrm{CI}]$ was significantly lower in the paternal $\mathrm{SCH}$ group than the paternal euthyroidism group (0.34 [0.27-0.42] vs. $0.43[0.40-0.45], p=0.034)$ in the IVF cycles. Of the 396 ICSI cycles, 366 and 30 men were characterized by euthyroidism and $\mathrm{SCH}$, respectively. The clinical pregnancy rate stratified by ICSI cycles in the paternal SCH group was $13 \%$ lower than in the paternal euthyroidism group, but this decrease was not statistically significant $(0.25$ vs. 0.38 , $p=0.15)$. Compared with the paternal euthyroidism group, the implantation rates were $5 \%$ and $9 \%$ lower in the paternal $\mathrm{SCH}$ group stratified by IVF and ICSI cycles, respectively (adjusted means: 0.25 vs. 0.30 and 0.14 vs. 0.23 ); however, these differences were not significant $(p=0.11$ and 0.07 , respectively). We found no significance differences for any of the other outcome measures, including the fertilization rate, good-embryo rate, blastocyst formation rate, and early miscarriage rate (all $p>0.05$ ) between the paternal $\mathrm{SCH}$ and euthyroidism groups (Supplementary Table S1).

Table 3. Associations Between Male Subclinical Hypothyroidism and In Vitro Fertilization Outcomes (Presented as Adjusted Value [Confidence Interval])

\begin{tabular}{|c|c|c|c|}
\hline Outcomes & Euthyroidism $(\mathrm{n}=2282)$ & $S C H(\mathrm{n}=229)$ & $\mathrm{p}$ \\
\hline \multicolumn{4}{|l|}{ Primary outcomes } \\
\hline Fertilization rate $^{\mathrm{a}}$ & $0.71[0.70-0.72]$ & $0.69[0.66-0.73]$ & 0.30 \\
\hline Clinical pregnancy rate ${ }^{b}$ & $0.42[0.40-0.45]$ & $0.32[0.26-0.40]$ & 0.009 \\
\hline \multicolumn{4}{|l|}{ Secondary outcomes } \\
\hline Good-quality embryo rate & $0.52[0.50-0.53]$ & $0.49[0.45-0.54]$ & 0.31 \\
\hline Blastocyst formation rate ${ }^{a}$ & $0.53[0.51-0.54]$ & $0.51[0.46-0.56]$ & 0.57 \\
\hline Implantation rate ${ }^{\mathrm{b}}$ & $0.29[0.27-0.31]$ & $0.24[0.19-0.29]$ & $\mathbf{0 . 0 3 7}$ \\
\hline Early miscarriage rate ${ }^{b}$ & $0.10[0.08-0.13]$ & $0.11[0.05-0.21]$ & 0.81 \\
\hline
\end{tabular}

Bold $p$-values indicate a significant difference.

${ }^{a}$ Adjusted for male age, BMI, TAI, smoking status, alcohol consumption, female age, BMI, TAI, primary/secondary infertility, infertility diagnosis, no. of aspirated oocytes, ovarian stimulation protocol, and IVF/ICSI insemination.

${ }^{\mathrm{b}}$ Adjusted for male age, BMI, TAI, smoking status, alcohol consumption, female age, BMI, TAI, primary/secondary infertility, infertility diagnosis, ovarian stimulation protocol, IVF/ICSI cycles, fresh/frozen cycles, no. of transferred embryos, and day of embryo transfer (day 3 or 5/6). ICSI, intracytoplasmic sperm injection; IVF, in vitro fertilization. 
Table 4. Associations Between Male Subclinical Hypothyroidism and In Vitro Fertilization Outcomes Stratified by Age (Presented as Adjusted Value [Confidence Interval])

\begin{tabular}{|c|c|c|c|c|c|c|}
\hline \multirow[b]{2}{*}{ Outcomes } & \multicolumn{3}{|c|}{$<35$ years $(\mathrm{n}=1365)$} & \multicolumn{3}{|c|}{$\geq 35 \operatorname{years}(\mathrm{n}=1146)$} \\
\hline & $\begin{array}{l}\text { Euthyroidism } \\
(\mathrm{n}=1256)\end{array}$ & $S C H(\mathrm{n}=109)$ & $\mathrm{p}$ & $\begin{array}{l}\text { Euthyroidism } \\
(\mathrm{n}=1026)\end{array}$ & $S C H(\mathrm{n}=120)$ & $\mathrm{p}$ \\
\hline Fertilization rate ${ }^{\mathrm{a}}$ & $0.71[0.69-0.72]$ & $0.69[0.66-0.74]$ & 0.58 & $0.72[0.70-0.74]$ & $0.70[0.65-0.75]$ & 0.42 \\
\hline Good-quality embryo rate ${ }^{a}$ & $0.51[0.49-0.53]$ & $0.48[0.43-0.54]$ & 0.34 & $0.53[0.51-0.55]$ & $0.52[0.45-0.59]$ & 0.72 \\
\hline Blastocyst formation rate ${ }^{a}$ & $0.54[0.52-0.56]$ & $0.53[0.48-0.59]$ & 0.84 & $0.48[0.46-0.51]$ & $0.46[0.37-0.56]$ & 0.58 \\
\hline Clinical pregnancy rate & $0.49[0.46-0.52]$ & $0.45[0.35-0.54]$ & 0.39 & $0.31[0.27-0.35]$ & $0.18[0.11-0.29]$ & 0.009 \\
\hline Implantation rate ${ }^{\mathrm{b}}$ & $0.35[0.32-0.37]$ & $0.32[0.25-0.40]$ & 0.45 & $0.21[0.18-0.23]$ & $0.13[0.08-0.21]$ & 0.022 \\
\hline Early miscarriage rate ${ }^{\mathrm{b}}$ & $0.08[0.06-0.11]$ & $0.08[0.03-0.19]$ & 0.90 & $0.15[0.11-0.21]$ & $0.18[0.05-0.46]$ & 0.81 \\
\hline
\end{tabular}

Bold $p$-values indicate a significant difference.

${ }^{a}$ Adjusted for male age, BMI, TAI, smoking status, alcohol consumption, female age, BMI, TAI, primary/secondary infertility, infertility diagnosis, no. of aspirated oocytes, ovarian stimulation protocol, and IVF/ICSI insemination.

${ }^{\mathrm{b}}$ Adjusted for male age, BMI, TAI, smoking status, alcohol consumption, female age, BMI, TAI, primary/secondary infertility, infertility diagnosis, ovarian stimulation protocol, IVF/ICSI cycles, fresh/frozen cycles, no. of transferred embryos, and day of embryo transfer (day 3 or $5 / 6)$.

\section{Associations between male $\mathrm{SCH}$ and/or TAl and $A R T$ outcomes}

To investigate whether TAI affects the association between paternal SCH and ART outcomes, we divided the couples into four groups based on paternal euthyroidism $(n=2150)$, isolated SCH $(n=197)$, isolated TAI $(n=132)$, and SCH+TAI $(n=32)$. After adjusting for the potential confounders, we found no significant differences in the fertilization rate, good-embryo rate, or blastocyst formation rate among these four groups $(p=0.08,0.61$, and 0.75 , respectively). The clinical outcome measures varied significantly between the groups: we detected the highest adjusted clinical pregnancy rate $[\mathrm{CI}]$ in the euthyroidism group $(0.43$ $[0.40-0.45])$ and the lowest rate in the SCH+TAI group $(0.20$ [0.08-0.41]). We also found the highest adjusted implantation rate $[\mathrm{CI}]$ in the euthyroidism group $(0.30[0.28-0.31])$ and the lowest rate in the SCH+TAI group (0.14 [0.05-0.38]). When taking the euthyroidism group as a reference, the other three groups all had a significantly lower clinical pregnancy rate $(p=0.038,0.043$, and 0.007 , respectively). The implantation rate in the isolated $\mathrm{SCH}$ group showed a slight but nonsignificant decrease compared with the euthyroidism group $(p=0.06)$; the implantation rates in the isolated TAI and SCH+TAI groups were all significantly lower compared with the euthyroidism group $(p=0.048$ and 0.036 , respectively) (Table 5).

\section{Associations between male TSH levels and IVF/ICSI outcomes}

To further investigate the associations between TSH levels and IVF/ICSI outcomes, we divided the couples into three groups according to male TSH levels: euthyroidism $(n=2282)$, TSH $4.2-8.0 \mathrm{mIU} / \mathrm{mL}(n=200)$, and TSH $>8.0$ $\mathrm{mIU} / \mathrm{mL}(n=29)$. The results showed that the adjusted fertilization rate and good-embryo rate among these three groups were not significantly different. However, the blastocyst formation rate in men with $\mathrm{TSH}>8.0 \mathrm{mIU} / \mathrm{mL}$ was

Table 5. In Vitro Fertilization Outcomes Between Euthyroid Men and Men with Subclinical Hypothyroidism and/or Thyroid Autoimmunity (Presented as Adjusted Value [Confidence Interval])

\begin{tabular}{lcccc}
\hline Outcomes & $\begin{array}{c}\text { Euthyroidism } \\
(\mathrm{n}=2150)\end{array}$ & $\begin{array}{c}\text { Isolated } S C H \\
(\mathrm{n}=197)\end{array}$ & $\begin{array}{c}\text { Isolated TPO-Ab } \\
\text { positivity }(\mathrm{n}=132)\end{array}$ & $\begin{array}{c}S C H+T P O-A b \\
\text { positivity }(\mathrm{n}=32)\end{array}$ \\
\hline Fertilization rate $^{\mathrm{a}}$ & $0.71[0.70-0.72]$ & $0.68[0.65-0.72]$ & $0.68[0.64-0.72]$ & $0.76[0.68-0.85]$ \\
$p$ & Reference & 0.09 & 0.10 & 0.27 \\
Good-quality embryo rate $^{\mathrm{a}}$ & $0.53[0.50-0.53]$ & $0.49[0.45-0.54]$ & $0.54[0.48-0.60]$ & $0.53[0.43-0.65]$ \\
$p$ & Reference & 0.30 & 0.47 & 0.79 \\
Blastocyst formation rate $^{\mathrm{a}}$ & $0.52[0.51-0.54]$ & $0.51[0.46-0.56]$ & $0.55[0.49-0.61]$ & $0.55[0.42-0.72]$ \\
$p$ & Reference & 0.54 & 0.42 & 0.72 \\
Clinical pregnancy rate $^{\mathrm{b}}$ & $0.43[0.40-0.45]$ & $0.34[0.27-0.42]$ & $0.33[0.25-0.43]$ & $0.20[0.08-0.41]$ \\
$p$ & Reference & $\mathbf{0 . 0 3 8}$ & $\mathbf{0 . 0 4 3}$ & $\mathbf{0 . 0 0 7}$ \\
Implantation rate $^{\mathrm{b}}$ & $0.30[.28-0.31]$ & $0.25[0.20-0.30]$ & $0.23[0.17-0.30]$ & $0.14[0.05-0.38]$ \\
$p$ & Reference & 0.06 & $\mathbf{0 . 0 4 8}$ & $\mathbf{0 . 0 3 6}$ \\
Early miscarriage rate $^{\mathrm{b}}$ & $0.10[0.08-0.12]$ & $0.12[0.06-0.22]$ & $0.12[0.05-0.27]$ & $0.00[0.00-1.00]$ \\
$p$ & Reference & 0.79 & 0.71 & 0.99 \\
\hline
\end{tabular}

Bold $p$-values indicate a significant difference.

${ }^{a}$ Adjusted for male age, BMI, smoking status, alcohol consumption, female age, BMI, TAI, primary/secondary infertility, infertility diagnosis, no. of aspirated oocytes, ovarian stimulation protocol, and IVF/ICSI insemination.

${ }^{\mathrm{b}}$ Adjusted for male age, BMI, smoking status, alcohol consumption, female age, BMI, TAI, primary/secondary infertility, infertility diagnosis, ovarian stimulation protocol, IVF/ICSI cycles, fresh/frozen cycles, no. of transferred embryos, and day of embryo transfer (day 3 or 5/6).

TPO-Ab, thyroperoxidase antibody. 
significantly lower than that in euthyroid men (adjusted rate: 0.37 vs. $0.53, p=0.043$ ). Men with TSH $4.2-8.0 \mathrm{mIU} / \mathrm{mL}$ and $>8.0 \mathrm{mIU} / \mathrm{mL}$ had a significantly lower clinical pregnancy rate ( 0.34 and 0.21 , respectively) than that in euthyroid men (0.42) ( $p=0.038$ and 0.015 , respectively). Implantation rate in men with $\mathrm{TSH} 4.2-8.0 \mathrm{mIU} / \mathrm{mL}$ was nonsignificantly lower than that in euthyroid men (adjusted implantation rate 0.26 vs. $0.30, p=0.16$ ), whereas implantation rate in men with TSH $>8.0 \mathrm{mIU} / \mathrm{mL}$ was significantly lower than that in euthyroid men (adjusted implantation rate 0.12 vs. 0.30 , $p=0.02$ ) (Supplementary Table S2).

\section{Discussion}

To date, most studies have focused on the effects of maternal thyroid dysfunction on pregnancy outcomes. In contrast, very few studies have considered the potential effects of paternal thyroid dysfunction, particularly a mild condition such as $\mathrm{SCH}$, on pregnancy outcomes. To the best of our knowledge, this study constitutes the first evaluation of the potential effects of paternal SCH on ART clinical outcomes and the association between thyroid function parameters and ART clinical outcomes. The SCH prevalence in our cohort was $9.1 \%$. We observed nonsignificant decreases in the fertilization rate, good-quality embryo rate, and blastocyst formation rate in cycles involving men with $\mathrm{SCH}$ compared with couples involving men with euthyroidism. Nevertheless, paternal SCH was significantly associated with a decreased clinical pregnancy rate and implantation rate, but was not significantly associated with an early miscarriage rate.

Although $\mathrm{SCH}$ is only a mild thyroid dysfunction, this condition might reflect a relatively lower thyroid functional capacity that may become apparent in the future. Others have reported on the negative effects of hypothyroidism on male reproductive function $(7,26)$, and particularly on sperm concentration, motility, and morphology (27,28). Similar observations have been made in animal models (29). Very few studies, however, have focused on the effects of SCH on male reproduction. In 2001, Trummer et al. conducted a study based on 305 patients and found no association between thyroid dysfunction and semen parameters (30). Another study published in 2006 showed that the prevalence of thyroid dysfunction and autoimmunity is comparable between men with normal and men with abnormal semen characteristics (31). In 2015, Lotti et al. (32) conducted a study of 145 euthyroid men and 12 men with SCH and found no significant differences in sperm parameters or seminal fructose concentrations. We also found no significant differences in sperm parameters or DFI between euthyroid men and men with $\mathrm{SCH}$. We did observe a slightly higher DFI in men with $\mathrm{SCH}$ compared with euthyroid men (19.7\% vs. $18.7 \%)$.

Our results suggest that paternal $\mathrm{SCH}$ confers negative ART outcomes, although some of the differences in the outcome measurements were not statistically significant. As only a few studies have delineated the mechanistic links between thyroid dysfunction and spermatogenesis, we put forward the following proposals that might help explain the issues. First, animal studies have indicated that a hypothyroid state can affect sperm function (29). An increase in reactive oxygen species production was also observed in sperm isolated from hypothyroid animal models (33). As SCH reflects only a subtle deficiency in thyroid function, it is possible that
SCH exerts mild effects on sperm function and the subsequent embryo development. Second, DNA damage in sperm might be another critical factor that mediates poor $\mathrm{SCH}-$ induced ATR outcomes. Increased amounts of DNA damage were observed in the sperm of hypothyroid animal models $(33,34)$. A higher DFI has also been associated with lower embryo cleavage rates (35), resulting in a reduced potential to develop into blastocysts (36) and a decreased clinical pregnancy rate (37). The slight but nonsignificant increase in DFI in men with $\mathrm{SCH}$ included in this study might have contributed to the observed clinical outcomes. In addition, epigenetic alterations in the sperm might affect embryonic development. Indeed, a previous study based on thyroid rat models reported that THs regulated DNA methylation and histone modifications in astrocytes (38). Spermatozoa contain noncoding RNAs (such as microRNAs, small interfering RNAs, and piwi-interacting RNAs) that are transmitted to the oocyte (39-41). Animal studies have demonstrated that these noncoding RNAs have important roles in the epigenetic regulation of zygote development and the adult outcomes of the offspring (42). Despite these data, a human-based study has been absent from the literature.

We were interested to note that the differences observed in all laboratory outcomes (the fertilization rate, good-quality embryo rate, and blastocyst formation rate) did not significantly differ between the paternal $\mathrm{SCH}$ and euthyroidism groups, whereas the clinical outcomes did significantly differ. This observation can be explained by the fact that embryonic genome activation occurs only after the second cell division, that is, the four- to eight-cell stage (43), and thus is bound to affect only the later stages of embryo development.

Increasing evidence shows that advanced paternal age is associated with dysfunction of reproductive hormone synthesis and sperm production, decreased fecundability, adverse clinical pregnancy outcomes, and increased incidence of some birth defects (44). A recent study showed that paternal age had a $3 \%$ decrease in the odds of clinical pregnancy, a $4 \%$ decrease in the odds of live birth, a $3.1 \%$ decrease in the odds of embryo implantation, and a $5 \%$ increase in the odds of miscarriage with each year of age, in IVF/ICSI cycles, independent of female age (45). Increased DFI may be one of the explanations for the adverse clinical outcomes. The DFI increases with age $(46,47)$. In this study, the mean DFI in men aged $<35$ and $\geq 35$ years was $17.7 \%$ and $20.5 \%$, respectively. The percentages of men with a DFI $\geq 30 \%$ were $11.5 \%$ and $5.7 \%$ in men with $\mathrm{SCH}$ and men with euthyroidism, respectively. Aging is also recognized to be associated with more frequent germ cell and sperm DNA mutations (44) and excess reactive oxygen species (48); these pathologic alterations may affect male fertility. Interestingly, $\mathrm{SCH}$ was also reported to be associated with these above alterations, as reported in both human and animal models $(33,49)$, indicating that aging may play an additive effect on $\mathrm{SCH}$. This effect could account for the observed phenomenon that only differences in clinical outcomes (clinical pregnancy rate and implantation rate) in older men ( $\geq 35$ years) were statistically significant. Therefore, this study demonstrates that aging might amplify the impact of paternal $\mathrm{SCH}$ on pregnancy outcomes.

Our subgroup analysis showed that paternal SCH significantly affected the clinical pregnancy rate in IVF cycles, whereas for couples who had ICSI treatment, the influence of 
paternal SCH on clinical outcomes was not significant, although a lower clinical pregnancy rate and implantation rate were observed in the SCH group. Due to the very limited number of patients with SCH receiving ICSI treatment, the impact of paternal SCH on ICSI outcomes is uncertain, and future larger cohort studies are needed.

Like $\mathrm{SCH}$, TAI is also recognized as a mild thyroid dysfunction and might reflect a relatively low thyroid functional capacity that can become apparent in the future (6). This finding could account for the significant decrease in the clinical pregnancy rate and implantation rate in both paternal $\mathrm{SCH}$ and TAI groups compared with the euthyroidism group. Our findings suggest that SCH+TAI might further deteriorate thyroid function, thus leading to a further decrease in the probability to achieve clinical pregnancy (Table 5). The impact of paternal TAI on IVF/ICSI outcomes was different from maternal TAI. Busnelli et al. conducted a meta-analysis and showed that maternal TAI did not impact IVF/ICSI outcomes in terms of the likelihood of fertilization, implantation, and clinical pregnancy, whereas TAI may increase the risk of miscarriage and preterm birth and decrease the live birth rate (50). However, for couples receiving ICSI treatment, maternal TAI did not impact laboratory or clinical outcomes, as shown in another recently published metaanalysis by Poppe et al. (51). These results indicated that the impact of TAI on pregnancy outcomes may differ between patients who receive IVF and ICSI treatment. However, further studies are needed before drawing a firm conclusion.

Our study constitutes the first to investigate the association between paternal SCH and ART outcomes, and has several strengths. First, the application of strict inclusion and exclusion criteria ensures the reliability of our results. Second, thyroid function testing was conducted by only two highly trained technicians, to minimize the effects of interassay variability. Third, all embryos were cultured under the same conditions in the same laboratory; the embryologists graded the embryos using standard morphologic criteria and were blinded to the different conditions. Fourth, we adjusted for a series of potential confounders in the data analysis (including lifestyle habits and clinical characteristics) to avoid bias. Despite these strengths, however, some limitations should also be acknowledged. First, this study was based on a retrospective design; therefore, we could not establish a causal relationship. Second, female age in the SCH and euthyroidism groups was not well matched in this study, which may inevitably introduce a selection bias on clinical outcomes, although age was adjusted as a continuous variable in all regression models. It is recognized that female age is one of the most critical factors affecting ART outcomes (52), and therefore, age-matched patients in the $\mathrm{SCH}$ and euthyroidism groups may help eliminate the selection bias. Finally, the study participants were patients seeking fertility treatment; therefore, the results of this study might only reflect the effect of paternal SCH on ART outcomes. It remains unclear whether paternal $\mathrm{SCH}$ is associated with pregnancy outcomes in couples who conceive naturally.

In conclusion, we found that SCH affected $9.1 \%$ of men in our cohort. Paternal SCH was associated with significantly worse clinical outcomes in couples receiving ART treatment who had a male partner $\geq 35$ years old. We also identified a negative effect of TAI on clinical outcomes. Given the retrospective nature of this study, prospective studies and basic research are warranted to confirm our results and clarify the mechanisms underlying these associations, respectively. Randomized clinical trials might also provide high-quality evidence regarding the effects of LT4 supplementation on clinical outcomes following ART in couples with paternal $\mathrm{SCH}$.

\section{Acknowledgments}

We are grateful for the patients who participated in this study. We acknowledge the professional article editing services of Armstrong-Hilton Ltd.

\section{Author Disclosure Statement}

No competing financial interests exist.

\section{Funding Information}

This study was supported by the National Natural Science Foundation of China (No. 81801416, 81760269), the Technology \& Innovation Team of Reproduction and Genetics from Kunming Medical University (No. CXTD201708), the Scientific Funding from the First Affiliated Hospital of Kunming Medical University (No. 2017BS008), the Joint Fund of Science and Technology Department of Yunnan Province and Kunming Medical University under Grant (2017FE467-137); Yunnan Applied Basic Research Project under grant (2018FB126); and Personnel Training Program of Young and Middle-age Academic and Technical Leaders of Yunnan Province under grant (2017HB046).

\section{Supplementary Material}

Supplementary Table S1

Supplementary Table S2

\section{References}

1. Rao M, Wang H, Zhao S, Liu J, Wen Y, Wu Z, Yang Z, Su C, Su Z, Wang K, Tang L 2019 Subclinical hypothyroidism is associated with lower ovarian reserve in women aged 35 years or older. Thyroid 30:95-105.

2. Wang S, Teng WP, Li JX, Wang WW, Shan ZY 2012 Effects of maternal subclinical hypothyroidism on obstetrical outcomes during early pregnancy. J Endocrinol Invest 35:322-325.

3. Maraka S, Ospina NM, O'Keeffe DT, Espinosa De Ycaza AE, Gionfriddo MR, Erwin PJ, Coddington CC, 3rd, Stan MN, Murad MH, Montori VM 2016 Subclinical hypothyroidism in pregnancy: a systematic review and metaanalysis. Thyroid 26:580-590.

4. Chen S, Zhou X, Zhu H, Yang H, Gong F, Wang L, Zhang M, Jiang Y, Yan C, Li J, Wang Q, Zhang S, Pan H 2017 Preconception TSH and pregnancy outcomes: a populationbased cohort study in 184611 women. Clin Endocrinol (Oxf) 86:816-824.

5. Zhang Y, Wang H, Pan X, Teng W, Shan Z 2017 Patients with subclinical hypothyroidism before 20 weeks of pregnancy have a higher risk of miscarriage: a systematic review and meta-analysis. PLoS One 12:e0175708.

6. Rao M, Zeng Z, Zhou F, Wang H, Liu J, Wang R, Wen Y, Yang Z, Su C, Su Z, Zhao S, Tang L 2019 Effect of levothyroxine supplementation on pregnancy loss and preterm birth in women with subclinical hypothyroidism and 
thyroid autoimmunity: a systematic review and metaanalysis. Hum Reprod Update 25:344-361.

7. Krassas GE, Poppe K, Glinoer D 2010 Thyroid function and human reproductive health. Endocr Rev 31:702-755.

8. Jannini EA, Ulisse S, D'Armiento M 1995 Thyroid hormone and male gonadal function. Endocr Rev 16:443-459.

9. Jannini EA, Crescenzi A, Rucci N, Screponi E, Carosa E, de Matteis A, Macchia E, d'Amati G, D'Armiento M 2000 Ontogenetic pattern of thyroid hormone receptor expression in the human testis. J Clin Endocrinol Metab 85:3453-3457.

10. Wagner MS, Wajner SM, Maia AL 2008 The role of thyroid hormone in testicular development and function. J Endocrinol 199:351-365.

11. La Vignera S, Vita R, Condorelli RA, Mongioi LM, Presti S, Benvenga S, Calogero AE 2017 Impact of thyroid disease on testicular function. Endocrine 58:397-407.

12. Mendeluk GR, Rosales M 2016 Thyroxin is useful to improve sperm motility. Int J Fertil Steril 10:208-214.

13. Du X, Ding T, Zhang H, Zhang C, Ma W, Zhong Y, Qu W, Zheng J, Liu Y, Li Z, Huang K, Deng S, Ma L, Yang J, Jiang J, Yang S, Huang J, Wu M, Fang L, Lu Y, Luo A, Wang S 2016 Age-specific normal reference range for serum anti-Mullerian hormone in healthy Chinese Han women: a nationwide population-based study. Reprod Sci 23:1019-1027.

14. Gode F, Gulekli B, Dogan E, Korhan P, Dogan S, Bige O, Cimrin D, Atabey N 2011 Influence of follicular fluid GDF9 and BMP15 on embryo quality. Fertil Steril 95: 2274-2278.

15. Ide A, Amino N, Kang S, Yoshioka W, Kudo T, Nishihara E, Ito M, Nakamura H, Miyauchi A 2014 Differentiation of postpartum Graves' thyrotoxicosis from postpartum destructive thyrotoxicosis using antithyrotropin receptor antibodies and thyroid blood flow. Thyroid 24:1027-1031.

16. Rao M, Zhou F, Tang L, Zeng Z, Hu S, Wang Y, Ke D, Cheng G, Xia W, Zhang L, Zhu C 2019 Follicular fluid humanin concentration is related to ovarian reserve markers and clinical pregnancy after IVF-ICSI: a pilot study. Reprod Biomed Online 38:108-117.

17. Rao M, Wu Z, Wen Y, Wang R, Zhao S, Tang L 2019 Humanin levels in human seminal plasma and spermatozoa are related to sperm quality. Andrology 7:859-866.

18. WHO 2010 WHO Laboratory Manual for the Examination and Processing of Human Semen, 5th ed. World Health Organization, Geneva.

19. Sadeghi S, Garcia-Molina A, Celma F, Valverde A, Fereidounfar S, Soler C 2016 Morphometric comparison by the ISAS((R)) CASA-DNAf system of two techniques for the evaluation of DNA fragmentation in human spermatozoa. Asian J Androl 18:835-839.

20. Fernandez JL, Muriel L, Goyanes V, Segrelles E, Gosalvez J, Enciso M, LaFromboise M, De Jonge C 2005 Simple determination of human sperm DNA fragmentation with an improved sperm chromatin dispersion test. Fertil Steril 84: 833-842.

21. Li Y, Li Y, Lai Q, Zhang H, Zhu G, Jin L, Yue J 2008 Comparison between a GnRH agonist and a GnRH antagonist protocol for the same patient undergoing IVF. J Huazhong Univ Sci Technolog Med Sci 28:618-620.

22. Xu B, Li Z, Zhang H, Jin L, Li Y, Ai J, Zhu G 2012 Serum progesterone level effects on the outcome of in vitro fertilization in patients with different ovarian response: an analysis of more than 10,000 cycles. Fertil Steril 97:13211327.e1321-e1324.
23. Duan L, Bao S, Li K, Teng X, Hong L, Zhao X 2017 Comparing the long-acting and short-acting forms of gonadotropin-releasing hormone agonists in the long protocol of IVF/ICSI Cycles: a retrospective study. J Obstet Gynaecol Res 43:1037-1042.

24. Yu S, Long H, Chang HY, Liu Y, Gao H, Zhu J, Quan X, Lyu Q, Kuang Y, Ai A 2018 New application of dydrogesterone as a part of a progestin-primed ovarian stimulation protocol for IVF: a randomized controlled trial including 516 first IVF/ICSI cycles. Hum Reprod 33:229_ 237.

25. Li J, Du M, Zhang Z, Guan Y, Wang X, Zhang X, Liu J, Pan Z, Wang B, Liu W 2018 Does a poor-quality embryo have an adverse impact on a good-quality embryo when transferred together? J Ovarian Res 11:78.

26. Rajender S, Monica MG, Walter L, Agarwal A 2011 Thyroid, spermatogenesis, and male infertility. Front Biosci (Elite Ed) 3:843-855.

27. Krassas GE, Papadopoulou F, Tziomalos K, Zeginiadou T, Pontikides N 2008 Hypothyroidism has an adverse effect on human spermatogenesis: a prospective, controlled study. Thyroid 18:1255-1259.

28. Nikoobakht MR, Aloosh M, Nikoobakht N, Mehrsay AR, Biniaz F, Karjalian MA 2012 The role of hypothyroidism in male infertility and erectile dysfunction. Urol J 9:405-409.

29. Romano RM, Gomes SN, Cardoso NC, Schiessl L, Romano MA, Oliveira CA 2017 New insights for male infertility revealed by alterations in spermatic function and differential testicular expression of thyroid-related genes. Endocrine 55:607-617.

30. Trummer H, Ramschak-Schwarzer S, Haas J, Habermann H, Pummer K, Leb G 2001 Thyroid hormones and thyroid antibodies in infertile males. Fertil Steril 76:254-257.

31. Poppe K, Glinoer D, Tournaye H, Maniewski U, Haentjens P, Velkeniers B 2006 Is systematic screening for thyroid disorders indicated in subfertile men? Eur $\mathbf{J}$ Endocrinol 154:363-366.

32. Lotti F, Maseroli E, Fralassi N, Degl'Innocenti S, Boni L, Baldi E, Maggi M 2016 Is thyroid hormones evaluation of clinical value in the work-up of males of infertile couples? Hum Reprod 31:518-529.

33. Alipour F, Jalali M, Nikravesh MR, Fazel A, Sankian M, Khordad E 2018 Assessment of sperm morphology, chromatin integrity, and catSper genes expression in hypothyroid mice. Acta Biol Hung 69:244-258.

34. Xian H, Wang F, Teng W, Yang D, Zhang M 2017 Thyroid hormone induce a p53-dependent DNA damage through PI3K/Akt activation in sperm. Gene 615:1-7.

35. Zini A 2011 Are sperm chromatin and DNA defects relevant in the clinic? Syst Biol Reprod Med 57:78-85.

36. Fatehi AN, Bevers MM, Schoevers E, Roelen BA, Colenbrander B, Gadella BM 2006 DNA damage in bovine sperm does not block fertilization and early embryonic development but induces apoptosis after the first cleavages. J Androl 27:176-188.

37. Simon L, Murphy K, Shamsi MB, Liu L, Emery B, Aston KI, Hotaling J, Carrell DT 2014 Paternal influence of sperm DNA integrity on early embryonic development. Hum Reprod 29:2402-2412.

38. Kumar P, Godbole NM, Chaturvedi CP, Singh RS, George N, Upadhyay A, Anjum B, Godbole MM, Sinha RA 2018 Mechanisms involved in epigenetic down-regulation of Gfap under maternal hypothyroidism. Biochem Biophys Res Commun 502:375-381. 
39. Yan W, Morozumi K, Zhang J, Ro S, Park C, Yanagimachi R 2008 Birth of mice after intracytoplasmic injection of single purified sperm nuclei and detection of messenger RNAs and MicroRNAs in the sperm nuclei. Biol Reprod 78:896-902.

40. Frost RJ, Hamra FK, Richardson JA, Qi X, Bassel-Duby R, Olson EN 2010 MOV10L1 is necessary for protection of spermatocytes against retrotransposons by Piwi-interacting RNAs. Proc Natl Acad Sci U S A 107:11847-11852.

41. Miller D 2007 Ensuring continuity of the paternal genome: potential roles for spermatozoal RNA in mammalian embryogenesis. Soc Reprod Fertil Suppl 65:373-389.

42. Chen Q, Yan M, Cao Z, Li X, Zhang Y, Shi J, Feng GH, Peng H, Zhang X, Zhang Y, Qian J, Duan E, Zhai Q, Zhou Q 2016 Sperm tsRNAs contribute to intergenerational inheritance of an acquired metabolic disorder. Science 351: 397-400.

43. Braude P, Bolton V, Moore S 1988 Human gene expression first occurs between the four- and eight-cell stages of preimplantation development. Nature 332:459-461.

44. Sartorius GA, Nieschlag E 2010 Paternal age and reproduction. Hum Reprod Update 16:65-79.

45. Horta F, Vollenhoven B, Healey M, Busija L, Catt S, Temple-Smith P 2019 Male ageing is negatively associated with the chance of live birth in IVF/ICSI cycles for idiopathic infertility. Hum Reprod 34:2523-2532.

46. Borges E, Jr., Zanetti BF, Setti AS, Braga D, Provenza RR, Iaconelli A, Jr. 2019 Sperm DNA fragmentation is correlated with poor embryo development, lower implantation rate, and higher miscarriage rate in reproductive cycles of non-male factor infertility. Fertil Steril 112:483-490.

47. Rosiak-Gill A, Gill K, Jakubik J, Fraczek M, Patorski L, Gaczarzewicz D, Kurzawa R, Kurpisz M, Piasecka M 2019 Age-related changes in human sperm DNA integrity. Aging (Albany NY) 11:5399-5411.

48. Selvaratnam JS, Robaire B 2016 Effects of aging and oxidative stress on spermatozoa of superoxide-dismutase 1- and catalase-null mice. Biol Reprod 95:60.

49. Novakovic TR, Dolicanin ZC, Djordjevic NZ 2018 Effects of maternal subclinical hypothyroidism on amniotic fluid cells oxidative status. Reprod Toxicol 78:97-101.

50. Busnelli A, Paffoni A, Fedele L, Somigliana E 2016 The impact of thyroid autoimmunity on IVF/ICSI outcome: a systematic review and meta-analysis. Hum Reprod Update 22:775-790.

51. Poppe K, Autin C, Veltri F, Kleynen P, Grabczan L, Rozenberg S, Ameye L 2018 Thyroid autoimmunity and intracytoplasmic sperm injection outcome: a systematic review and meta-analysis. J Clin Endocrinol Metab [Epub ahead of print]; DOI: 10.1210/jc.2017-02633.

52. Sunkara SK, Rittenberg V, Raine-Fenning N, Bhattacharya S, Zamora J, Coomarasamy A 2011 Association between the number of eggs and live birth in IVF treatment: an analysis of 400135 treatment cycles. Hum Reprod 26: $1768-1774$.

Address correspondence to:

Kunhua Wang, MD, PhD

The NHC Key Laboratory of Drug Addiction Medicine The First Affiliated Hospital of Kunming

Medical University

Kunming 650032

China

E-mail: kunhuawang1@163.com

Li Tang, MD, PhD

Department of Reproduction and Genetics

The First Affiliated Hospital of Kunming

Medical University

No. 295 Xichang Road

Kunming 650032

China

E-mail: tanglikm@163.com

Shuhua Zhao, PhD

Department of Reproduction and Genetics

The First Affiliated Hospital of Kunming

Medical University

No. 295 Xichang Road

Kunming 650032

China

E-mail: kmshh1982@126.com 